\title{
Modified Korteweg-de Vries (mK-dV) Equation Describing Dust-ion-acoustic Solitary Waves in an Unmagnetized Dusty Plasma with Trapped Negative Ions
}

\author{
Obaydur Rahman and Md. Masum Haider \\ Department of Physics, Mawlana Bhashani Science and Technology University, Santosh, Tangail, Bangladesh \\ Email: armanphy203@gmail.com
}

\begin{abstract}
The effects of vortex-like (trapped) negative ion, Maxwellians electron, arbitrarily charged stationary dust, and cold mobile inertial positive ion have been theoretically investigated in the study of nonlinear propagation of dust-ion-acoustic (DIA) solitary waves (SWs) in an unmagnetized dusty electronegative plasma (DENP). The properties of small but finite amplitude DIASWs are studied by employing the reductive perturbation technique (RPT). It has been found that owing to the departure from the Maxwellian negative ion distribution to a vortex-like one, the dynamics of such DIASWs is governed by a modified Korteweg-de Vries (mK-dV) equation which admits solitary wave solution under certain conditions. The basic properties (speed, amplitude, width, etc.) of such DIASWs are found to be significantly modified by the effects of trapped negative ions and arbitrarily charged stationary dust particles. The results should be useful for understanding the nonlinear propagation of DIASWs in laboratory and space plasmas (e.g., Earth's magnetosphere, auroral region, heliospheric environments etc.).
\end{abstract}

Keywords: Dust-ion-acoustic waves, solitary waves, electronegative dusty plasma, trapped negative ions.

\section{Introduction}

Nowadays, electronegative plasmas i.e, plasmas with a significant amount of negative ions [1], [2], [3], [4], which are highly chemically reactive have attracted a great deal of interest [5], [6], [7], [8], [9], [10], [11], [12] because of their wide technological applications, e.g., semiconductor materials processing [13], synthesis of nanometerials [14], micro-electronic and photo-electronic industries [15] and they also plays vital role in many astrophysical plasmas e.g., D region of the ionosphere, the mesosphere, cometary tails, planetary rings, interplanetary space, interstellar medium, the evolution of the solar system from its solar nebula to its present form, etc. These electronegative plasmas are also found in the laboratory and plasmas technology such as low temperature physics (radio frequency), plasma discharge [16] and dusty crystal [17],[18].

However, the electronegative plasmas are not pure in general because it is contaminated by the dust particles e.g., solid impurities. Such impurities are not practically neutral but are charged, which means this dust particles may be either positively or negatively charged [19] by absorbing electrons, positive as well as negative ions [10], [20], [21], [22], [23], [24], [25], [26]. For this reason the electronegative plasmas are sometimes called dirty or dusty electronegative plasma (DENP) [10], [20], [21], [22], [23], [24], [25], [26]. Therefore, a DENP [27], [28] is roughly defined as a normal electron-ion plasma with an additional charged component of micron/sub-micron sized dust particles which increases the complexity of the plasma system. For this reason a dusty plasma is also referred to as a "complex plasma" [29].

Cousens et al. [30] have studied the nonlinear features of dust-acoustic (DA) waves in a three component plasma consisting of strongly coupled dust particle and Maxwellian electrons and ions. El-Labany et al. [31] have studied the nonlinear features of the DA waves which are the low frequency electrostatic waves in a dusty plasma system with mobile dust, where restoring force is provided by the thermal pressure of electrons and ions, and inertia is provided by the mass of dust. The nonlinear features of the DIA waves in DENP have been analyzed by a number of authors [32], [33], [34], they have predicted that negative ions in such electronegative plasmas obey Maxwellians distribution. Ghim and Hershkowitz have conclusively 
verified this prediction experimentally [35]. Kourakis et al. [36], Anowar et al. [37] and Moslem et al. [38] have studied the nonlinear features of the DIA which are also the low frequency electrostatic waves in a dusty plasma system with static dust in which restoring force is provided by the thermal pressure of electrons and inertia is provided by the mass of ion. The phase speed of such DIA waves is much smaller than the electron thermal speed but much larger than ion thermal speed, i.e, $v_{T i} \ll v_{p} \ll v_{T e}$.

Recently, motivated by the laboratory experiment [35] Mamun et al. [20], [39], [40] have considered a dusty electronegative plasma containing Maxwellian electrons, Maxwellian negative ions, cold mobile positive ions, and negatively charged stationary dust, and have examined the possibility for the formation of IA and DIA solitary waves and double layers in a DENP. In the work of Mamun et al. [39], they have considered unmagnetized dusty plasma consisting the electrons and ions which are isothermal. Some numerical investigations on linear and nonlinear DA waves show a significant amount of ions trapping in the wave potential, indicating that there is a departure from Boltzmann ion distribution and one encounter vortex-like ion distribution in phase space [41], [42], [43], [44].

Very recently Du et al. [45] have studied experimentally a new bursting $\mathrm{m}=1 / \mathrm{n}=1$ instability ( $\mathrm{m}, \mathrm{n}$ : poloidal and toroidal mode numbers) with rapid frequency chirping down in a helical plasma with intense perpendicular neutral beam which is destabilized by helically trapped energetic ions in a Large Halicon Device (LHD). It is also well known from [46], [47], [48], [49] that the electron and ion distribution functions can be significantly modified in the presence of large-amplitude waves that are excited by the two-stream instability [50]. Therefore, due to wave-particle interactions, the negative ions may not be isothermal (Maxwelliann), but they follow other distributions such as non-thermal [51] Kappa [52], or trapped distribution [46], [47], [53], which plays an important role in modifying basic features of nonlinear electrostatic structures [54], [55], [56], [57], [58] in many space and laboratory plasmas. Recently, Rahman and Mamun [59] have studied the nonlinear propagation of DIA solitary waves in an unmagnetized three components dusty plasma consisting of trapped electrons, cold mobile ions, and arbitrarily charged stationary dust. They have not considered negative ion which plays very important role in dusty plasma system. Therefore, in our present work, we have considered a four component DENP containing (i) positively charged mobile ions, (ii) non-isothermal negatively charged ion which may not have sufficient energy to overcome the potential, as a result it is trapped and following vortex-like distribution, (iii) Maxwellian electrons and (iv) arbitrarily charged stationary dust. We have studied the basic properties (phase speed, amplitude, width) of the DIA solitary waves.

The manuscript is organized as follows. The governing equations for a small but finite amplitude DIA SWs in an unmagnetized collisionless dusty plasma with vortex-like negative ions are presented in section 2. The well known mK-dV equation is derived by employing the reductive perturbation method in section 3 . The solitary wave solution of this $\mathrm{mK}-\mathrm{dV}$ equation is derived in section 4 . The numerical analysis of this work is presented in section 5. Finally, a brief summary of our results is discussed in section 6 .

\section{Governing Equations}

A collisionless unmagnetized four components DENP containing Maxwellian electrons, trapped negative ions, cold mobile positive ions, and arbitrarily charged stationary dust is considered. Thus, at equilibrium, $n_{p 0}-n_{e 0}-n_{n 0}+j Z_{d} n_{d 0}=0$, where $n_{p 0}, n_{e 0}, n_{n 0}$, and $n_{d 0}$ are, respectively, positive ion, electron, negative ion, and dust number density at equilibrium. $Z_{d}$ is the number of electrons residing onto the surface of a stationary dust, and $j=+1(-1)$ for positively (negatively) charged dust. We are interested in examining the nonlinear propagation of a low phase speed (in comparison with electron and negative ion thermal speeds), long wavelength (in comparison with Debye length) perturbation mode on the time scale of the DIA waves. The time scale of the DIA waves is much faster than the dust plasma period so that dust can be assumed stationary. The nonlinear dynamics of the low frequency electrostatic perturbation mode in such a DENP is described by

$$
\begin{aligned}
& \frac{\partial n_{p}}{\partial t}+\frac{\partial}{\partial x}\left(n_{p} u_{p}\right)=0 \\
& \frac{\partial u_{p}}{\partial t}+u_{p} \frac{\partial u_{p}}{\partial x}=-\frac{\partial \phi}{\partial x} \\
& \frac{\partial^{2} \phi}{\partial x^{2}}=\mu_{e} n_{e}-n_{p}+\mu_{n} n_{n}-j \mu_{d}
\end{aligned}
$$


where $n_{p}\left(n_{n}\right)$ is the positive (negative) ion number density normalized by $n_{p 0}\left(n_{n o}\right), u_{p}$ is the positive ion fluid speed normalized by the positive ion-acoustic speed $C_{p}=\left(k_{B} T_{e} / m_{p}\right)^{1 / 2}$, $\phi$ is the electrostatic wave potential normalized by $k_{B} T_{e} / e, \mu_{n}=\left(1-\mu_{e}+j \mu_{d}\right), \mu_{d}=Z_{d} n_{d 0} / n_{p 0}, \mu_{e}=n_{e 0} / n_{p 0}$, and $m_{p}$ is the mass of positive ion. The time variable $t$ is normalized by the positive ion plasma period $\omega_{p p}^{-1}$ and the space variable is normalized by $\lambda_{D}$.

The inertialess negative ion have a non-Maxwellians characteristics, described by a vortex-like velocity distribution which leads to a negative ion number density of the form [46], [47], [53], [55], [58], [60],

$$
n_{n}=1+\alpha \phi-\frac{4\left(1-\beta_{n}\right)}{3 \sqrt{\pi}} \alpha^{\frac{3}{2}} \phi^{\frac{3}{2}}+\frac{1}{2} \alpha^{2} \phi^{2}
$$

where $\alpha=Z_{n} T_{e} / T_{n}, T_{e}\left(T_{n}\right)$ is the electron (negative ion) temperature. $\beta_{n}$ is a parameter which determines the number of trapped negative ions [61], [62] and it is defined as the ratio of the free negative ion temperature $T_{i f}$ to the trapped negative ion temperature $T_{i t}$. We note that $\beta_{n}=1\left(\beta_{n}=0\right)$ represents a Maxwellian (flat-topped) negative ion distribution, whereas $\beta_{n}<0$ represents a vortex-like excavated trapped negative ion distribution. We are interested in $\beta_{n}<0$. On the other hand, the inertialess electron have a Maxwellian characteristics described by a Maxwellians Boltzmann velocity distribution which leads to a electron number density of the form

$$
n_{e}=e^{\phi}=1+\phi+\frac{1}{2} \phi^{2}
$$

\section{Derivation of Modified K-dV Equation}

To investigate the behavior of the small, but finite amplitude electrostatic DIAWs in the DENP under consideration, we employ the standard reductive perturbation method (RPM) [63]. The RPM is a very powerful way of deriving simplified models describing nonlinear wave propagation and interaction and was first introduced around 50 years ago by Washimi and Taniuti [63] to the study of ion-acoustic waves. Later, many world renounced scientists [19], [20], [21], [22], [46], [47], [53], [63], [64], [65], [66], [67], [68] worked using this method. The most natural approach, which is also the most affordable technically, is to use a perturbation method. Typically the fist order is the linear approximation and then the slowly varying envelope approximation. It can thus be qualified as "weakly nonlinear", in accordance with the terminology of mathematicians. However, many phenomena, although they are weakly nonlinear in the sense that the frequency and so on still have a meaning, diverge in their results from the linear situation. This is due to the fact that small modifications of an evolution law can yield large variations, if the evolution time is long enough. In this frame, the multiscale analysis is fully justified: it is a perturbative approach, whose first order is a linear approximation, that allows us to write small corrections valid for the same evolution time as in the linear approximation, but their cumulative effect lasts for a very long evolution. This method rescales both space and time in the governing equations of the system to introduce space and time variables, which are appropriate for the description of long-wavelength phenomena. According to this method, the independent variables are stretched as [46], [47], [53], [63]

$$
\left.\begin{array}{l}
\xi=\epsilon^{1 / 4}\left(x-v_{p} t\right) \\
\tau=\epsilon^{3 / 4} t
\end{array}\right\}
$$

where $\epsilon$ is a small dimensionless expansion parameter measuring the strength of nonlinearity and $v_{p}$ is the unknown phase speed (to be determined later) normalized by the positive ion-acoustic speed $\left(C_{p}\right)$. Furthermore, we can expand the physical quantities appearing in the basic equations (1-5) which are expanded as power series in $\epsilon$ about their equilibrium values [53], [64], [65], viz.

$$
\left.\begin{array}{l}
n_{p}=1+\epsilon n_{p}^{(1)}+\epsilon^{\frac{3}{2}} n_{p}^{(2)}+\cdots \\
u_{p}=0+\epsilon u_{p}^{(1)}+\epsilon^{\frac{3}{2}} u_{p}^{(2)}+\cdots \\
\phi=0+\epsilon \phi^{(1)}+\epsilon^{\frac{3}{2}} \phi^{(2)}+\cdots
\end{array}\right\}
$$


Now, using Eqs. (4)-(7) into Eqs. (1)-(3) one can obtain the first order continuity equation, momentum equation and Poisson's equation which, after simplification, yield

$$
\begin{aligned}
& n_{p}^{(1)}=\frac{\phi^{(1)}}{v_{p}^{2}} \\
& u_{p}^{(1)}=\frac{\phi^{(1)}}{v_{p}} \\
& v_{p}=\frac{1}{\sqrt{\mu_{e}+\alpha \mu_{n}}}
\end{aligned}
$$

Equation (10) represents the linear dispersion relation for DIA waves. Putting the values of Eqs. (4)-(10) into Eqs. (1)-(3), we obtain the next higher order equations,

$$
\begin{gathered}
\frac{\partial n_{p}^{(1)}}{\partial \tau}-v_{p} \frac{\partial n_{p}^{(2)}}{\partial \xi}+\frac{\partial u_{p}^{(2)}}{\partial \xi}=0 \\
\frac{\partial u_{p}^{(1)}}{\partial \tau}-v_{p} \frac{\partial u_{p}^{(2)}}{\partial \xi}+\frac{\partial \phi^{(2)}}{\partial \xi}=0 \\
\frac{\partial^{2} \phi^{(1)}}{\partial \xi^{2}}=\frac{\phi^{(2)}}{v_{p}^{2}}-\frac{4\left(1-\beta_{n}\right)}{3 \sqrt{\pi}} \mu_{n} \alpha^{3 / 2}\left[\phi^{(1)}\right]^{\frac{3}{2}}-n_{p}^{(2)}
\end{gathered}
$$

Now, Solving this system with the help of Eqs. (8)-(9) we can easily eliminate the second order perturbed quantities $n^{(2)}, u^{(2)}$ and $\phi^{(2)}$, and we finally obtain the mK-dV equations as

$$
\frac{\partial \phi^{(1)}}{\partial \tau}+A \sqrt{\phi^{(1)}} \frac{\partial \phi^{(1)}}{\partial \xi}+B \frac{\partial^{3} \phi^{(1)}}{\partial \xi^{3}}=0
$$

where,

$$
\begin{aligned}
& A=\frac{\left(1-\beta_{n}\right) \mu_{n} v_{p}^{3}}{\sqrt{\pi}} \alpha^{\frac{3}{2}} \\
& B=\frac{v_{p}^{3}}{2} .
\end{aligned}
$$

Equation (14) is a mK-dV equation, exhibiting a weakly nonlinearity, smaller width, and larger propagation velocity of the nonlinear wave.

\section{SW Solution of mK-dV Equation}

The stationary solution of this mK-dV equation can be obtained by transforming the independent variables $\xi$ and $\tau$ to $X=\xi-u_{0} \tau, \tau=\tau$, where $u_{0}$ is a constant velocity.

$$
\begin{aligned}
\frac{\partial}{\partial \tau} & =\frac{\partial}{\partial \tau}-u_{0} \frac{\partial}{\partial X} \\
\frac{\partial}{\partial \xi} & =\frac{\partial}{\partial X}
\end{aligned}
$$

Now substituting Eqs. (17)-(18) into Eq. (14) we get

$$
\frac{\partial \phi^{(1)}}{\partial \tau}-u_{0} \frac{\partial \phi^{(1)}}{\partial X}+A \sqrt{\phi^{(1)}} \frac{\partial \phi^{(1)}}{\partial X}+B \frac{\partial^{3} \phi^{(1)}}{\partial X^{3}}=0
$$

For steady state condition $\left(\partial \phi^{(1)} / \partial \tau \rightarrow 0\right)$ Eq. (19) reduces to

$$
-u_{0} \phi^{(1)}+\frac{2}{3} A \phi^{(1)^{3 / 2}}+B \frac{\mathrm{d}^{2} \phi^{(1)}}{\mathrm{d} X^{2}}=C_{1}
$$


where $C_{1}$ is an integration constant. Now under appropriate boundary conditions, viz. $\phi^{(1)} \rightarrow 0$ and $d^{2} \phi^{(1)} / d X^{2} \rightarrow 0$ at $X \rightarrow \pm \infty$, one can find $C_{1}$ as $C_{1}=0$. So, the above equation becomes

$$
\frac{\mathrm{d}^{2} \phi^{(1)}}{\mathrm{d} X^{2}}=\frac{1}{B}\left(u_{0} \phi^{(1)}-\frac{2}{3} A \phi^{(1)^{3 / 2}}\right)
$$

Now, multiplying both sides of the above equation by $d \phi^{(1)} / d X$, one gets

$$
\Rightarrow\left(\frac{\mathrm{d} \phi^{(1)}}{\mathrm{d} X}\right)^{2}=\frac{u_{0}}{B} \phi^{(1)^{2}}\left(1-\frac{8 A}{15 u_{0}} \phi^{(1)^{1 / 2}}\right)+C_{2}
$$

where $C_{2}$ is an another integration constant. Under the boundary conditions, $\phi^{(1)} \rightarrow 0$ and $d \phi^{(1)} / d X \rightarrow 0$ at $X \rightarrow \pm \infty$, one can express $C_{2}$ as $C_{2}=0$. So, the above equation becomes

$$
\frac{\mathrm{d} \phi^{(1)}}{\mathrm{d} X}=\phi^{(1)} \sqrt{\frac{u_{0}}{B}} \sqrt{1-\frac{8 A}{15 u_{0}} \phi^{(1)^{1 / 2}}}
$$

We now let

$$
\begin{aligned}
& \sqrt{1-\frac{8 A}{15 u_{0}} \phi^{(1)^{1 / 2}}}=Y \\
\Rightarrow & \phi^{(1)}=\left(\frac{15 u_{0}}{8 A}\right)^{2}\left(1-Y^{2}\right)^{2} \\
\Rightarrow & \frac{\mathrm{d} \phi^{(1)}}{\mathrm{d} X}=-4\left(\frac{15 u_{0}}{8 A}\right)^{2} Y\left(1-Y^{2}\right) \frac{\mathrm{d} Y}{\mathrm{~d} X}
\end{aligned}
$$

Now, substituting Eqs. (24)-(25) into Eq. (23), one obtains

$$
Y=\frac{\left(1-e^{X \sqrt{\frac{u_{0}}{4 B}}}\right)}{1+e^{X \sqrt{\frac{u_{0}}{4 B}}}}
$$

Now, substituting Eq. (26) into Eq. (24), one obtains

$$
\phi^{(1)}=\phi_{m} \operatorname{sech}^{4}\left[\frac{\left(\xi-u_{0} \tau\right)}{\Delta}\right]
$$

Eq. (27) is the stationary SWs solution of the mK-dV equation, where, $\phi_{m}=\left(\frac{15 u_{0}}{8 A}\right)^{2}$ and $\Delta=\sqrt{\frac{16 B}{u_{0}}}$ are the amplitude and the width of the SWs respectively.

\section{$5 \quad$ Numerical Analysis}

Figures 1 and 2 show the variation of the phase speed $v_{p}$ of SWs with $\mu_{d}$. From figure 1 , it has been found that the phase speed of the SWs becomes slower if the density of positively charged dust particles is increased in a dusty plasma system. On the other hand the phase speed of this SWs becomes faster when the density of negatively charged dust particles is increased in that system which is shown in figure 2. Figures 3-7 show the variation of the amplitude of solitary wave potential $\phi_{m}$ with $\alpha, \beta_{n}, \mu_{d}$ and $\mu_{e}$, respectively. Figure 3 indicates that the SWs become flat shape when the thermal pressure of electrons is increased and the polarity of dust particles have little effect on the SW. But when increasing the thermal pressure of negative ions the solitary structure becomes more spike. Figure 4 shows that the amplitude of SWs increases for both positively as well as negatively charged dust particles when increasing the value of trapping parameter $\beta_{n}$ but the amplitude is slightly greater for positively charged dust than for negatively charged dust. Figure 5 shows that, in the case of positively charged dust particles the potential of the SWs increases linearly when increasing the value of positive dust particles density. On the other hand, 


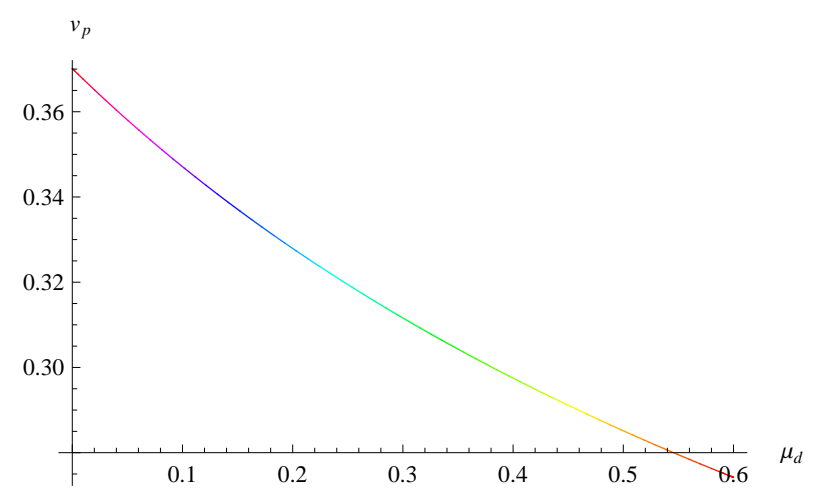

Figure 1. (Color online) The variation of the phase speed $\left(v_{p}\right)$ of SWs with $\mu_{d}$ for $j=+1, \mu_{e}=0.3$ and $\alpha=10$.

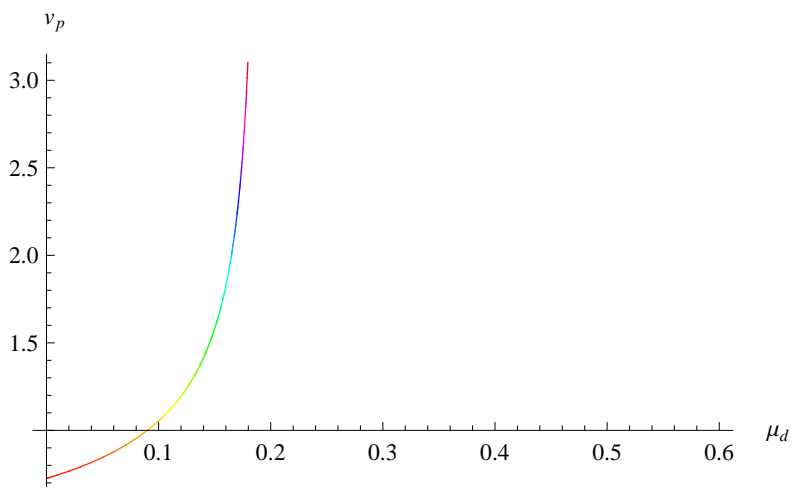

Figure 2. (Color online) The variation of the phase speed $\left(v_{p}\right)$ of SWs with $\mu_{d}$ for $j=-1, \mu_{e}=0.3$ and $\alpha=10$.

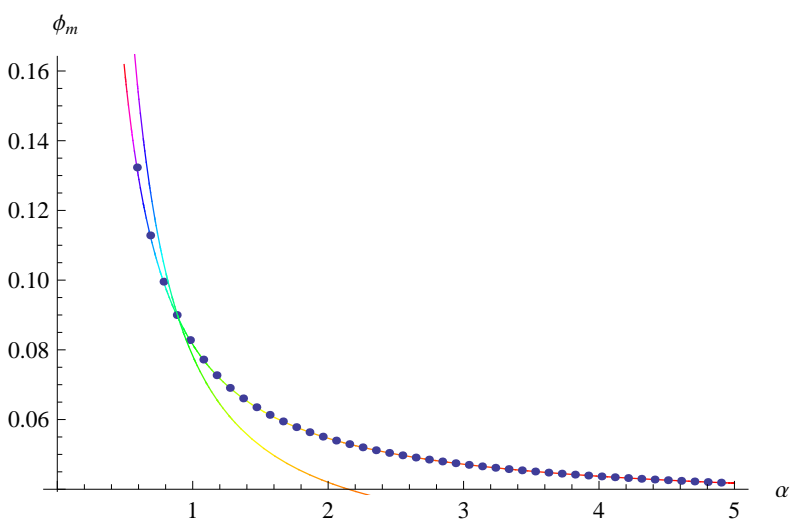

Figure 3. (Color online) The variation of the amplitude $\left(\phi_{m}\right)$ of SWs with $\alpha$ for $j=1(j=-1)$ dotted curve (solid curve), $u_{0}=0.1, \mu_{e}=0.3, \mu_{d}=0.2$, and $\beta_{n}=-0.7$.

the SWs potential decreases linearly with dust particle density $\mu_{d}$ when dust particles are negatively charged which is shown in figure 6 . From figure 7 , it has been seen that the amplitude decreases with the increase of the value of $\mu_{e}$ no matter dust particle is positive or negative but the amplitude is slightly larger for positively charged dust than for negatively charged dust. Figure 8 indicates that the width increases with the increase of the value of the density of electrons no matter dust particles are positive or negative but the width is slightly lower when the dust particles are positively charged. That means increasing the electron number density makes the solitary structure more flat shape. Figures 9 and 10 show the variation of the width $\Delta$ of solitary wave with $\mu_{d}$ for $j=1(-1)$ figure 9 (figure 10). From this 


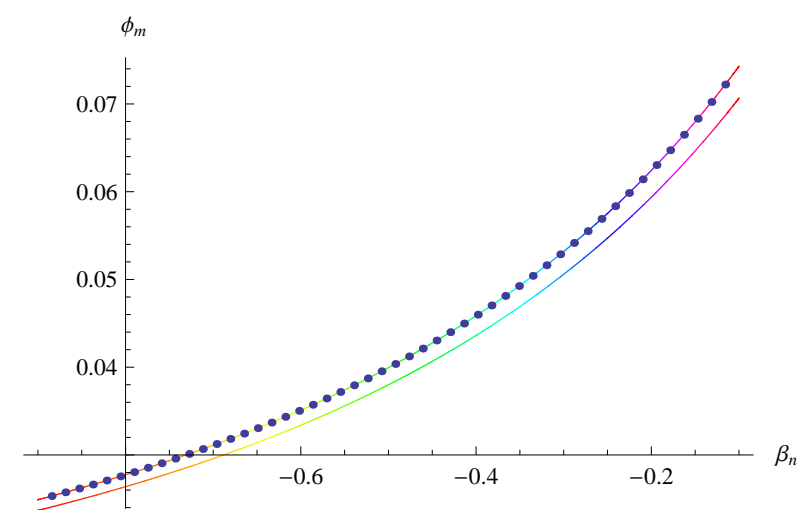

Figure 4. (Color online) The variation of the amplitude $\left(\phi_{m}\right)$ of SWs with $\beta_{n}$ for $j=1(j=-1)$ dotted curve (solid curve), $u_{0}=0.1, \mu_{e}=0.3, \mu_{d}=0.02$, and $\alpha=10$.

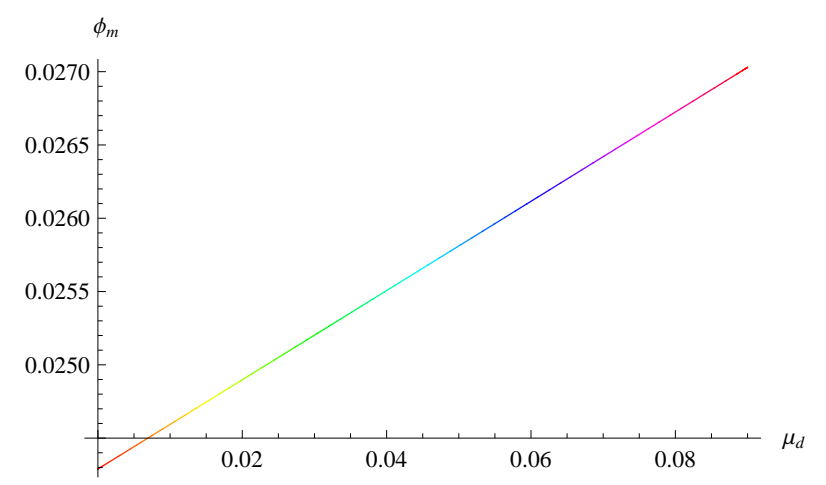

Figure 5. (Color online) The variation of the amplitude $\left(\phi_{m}\right)$ of SWs with $\mu_{d}$ for $j=+1, u_{0}=0.1, \mu_{e}=0.3$, $\alpha=10$, and $\beta_{n}=-0.9$.

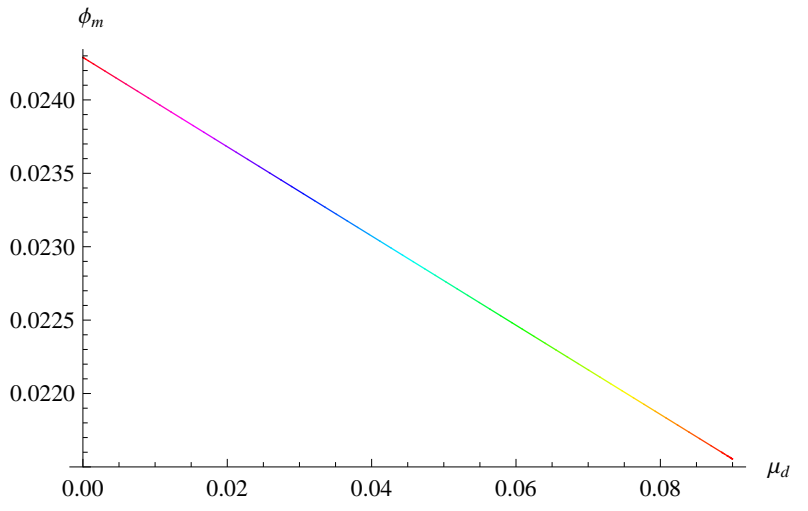

Figure 6. (Color online) The variation of the amplitude $\left(\phi_{m}\right)$ of SWs with $\mu_{d}$ for $j=-1, u_{0}=0.1, \mu_{e}=0.3$, $\alpha=10$, and $\beta_{n}=-0.9$.

figure it is clear that the width decreases with the increase of the number density of positively charged dust but it increases for the number density of negatively charged dust. 


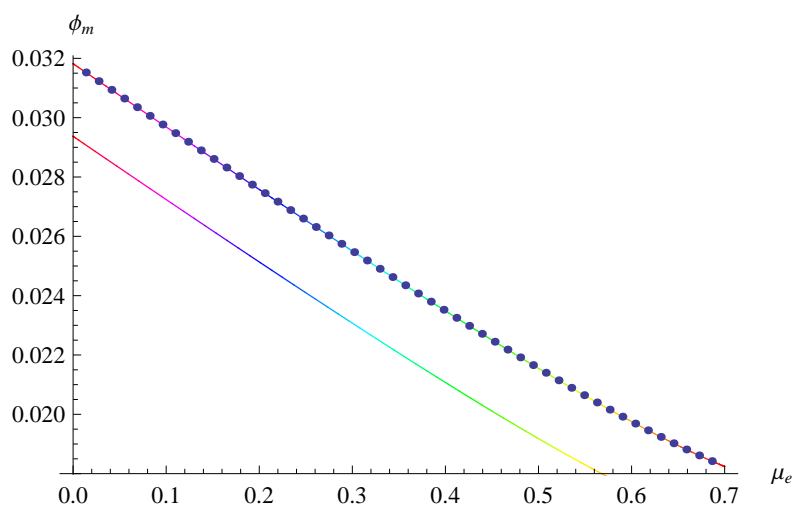

Figure 7. (Color online) The variation of the amplitude $\left(\phi_{m}\right)$ of SWs with $\mu_{e}$ for $j=1(j=-1)$ dotted curve (solid curve), $u_{0}=0.1, \mu_{d}=0.04, \alpha=10$, and $\beta_{n}=-0.9$.

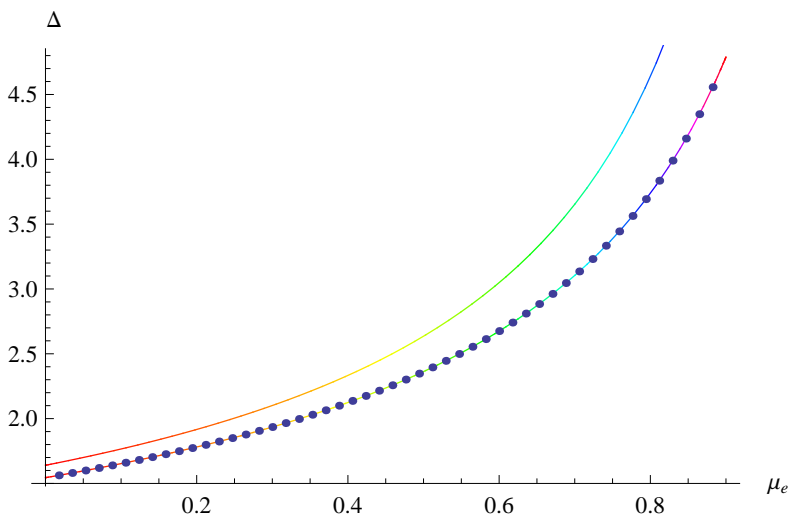

Figure 8. (Color online) The variation of the width $(\Delta)$ of SWs with $\mu_{e}$ for $u_{0}=0.1, j=1(j=-1)$ dotted curve (solid curve), $\alpha=10$, and $\mu_{d}=0.04$.

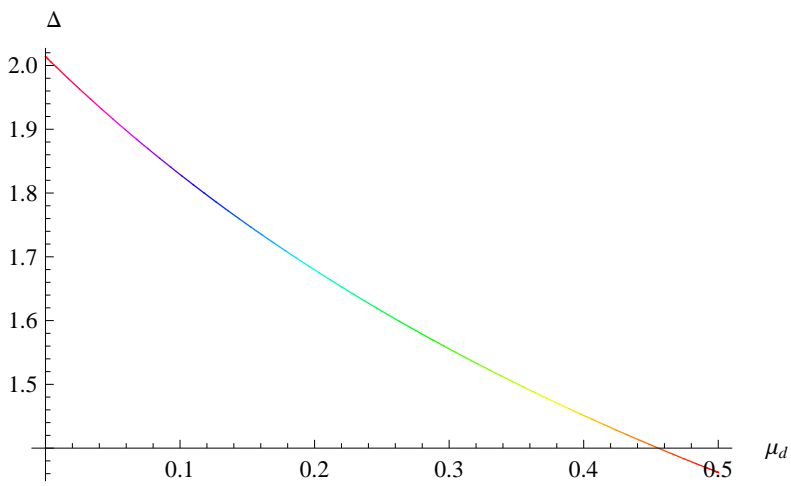

Figure 9. (Color online) The variation of the width $(\Delta)$ of SWs with $\mu_{d}$ for $u_{0}=0.1, j=1, \alpha=10$, and $\mu_{e}=0.3$.

\section{Discussion}

We have considered an unmagnetized four components DENP system consisting of electrons which obey Maxwellian distribution, negative ions satisfying vortex-like distribution, cold mobile positive ions, and arbitrarily charged stationary dust, and have studied the DIASWs by deriving mK-dV equation. It has been observed that the effect of dissipation in this case is negligible in comparison with those of the nonlinearity and dispersion. The basic features (phase speed, amplitude, and width) of such DIASWs are 


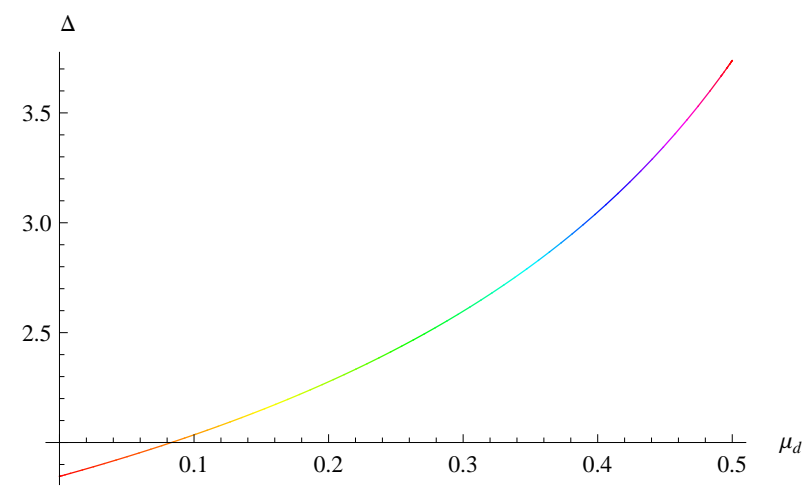

Figure 10. (Color online) The variation of the width $(\Delta)$ of SWs with $\mu_{d}$ for $u_{0}=0.1, j=-1, \alpha=10$, and $\mu_{e}=0.2$.

significantly modified by the presence of trapped negative ions, and arbitrarily charged stationary dust particles. The results which have been obtained from this investigation can be summarized as follows:

1. Dusty plasma system, whose constituents are cold mobile positive ions, Maxwellian electrons, trapped negative ions, and arbitrarily charged stationary dust of different constant temperatures, is found to support SWs associated with the non-linear DIA waves.

2. In the presence of the trapped negative ions distribution, the dynamics of weakly dispersive non-linear DIA waves are governed by the $\mathrm{mK}-\mathrm{dV}$ equation instead of $\mathrm{K}-\mathrm{dV}$ equation. Thus, the potential polarity of the DIA solitary waves in our dusty plasma is different from the usual IA solitary waves in an electron-ion plasma.

3. The profile of this wave can be represented in the form of $\operatorname{sech}^{4}(z / \Delta)$, instead of $\operatorname{sech}^{2}(z / \Delta)$ which is the stationary solution of the standard $\mathrm{K}-\mathrm{dV}$ equation for isothermal ions.

4. The dusty plasma system under consideration supports the DIA solitary waves that are associated with positive potential only. The fixed polarity of the potential structures is due to the effect of trapped negative ion distribution.

5. It has been found that the effect of trapped negative ion gives rise to a stronger nonlinearity (i.e., we have solitary structures with larger amplitude, smaller width, and higher propagation velocity).

6. From these above figures 1-2, it has been also found that the phase speed of the solitary wave increases with the increase of the values of $\mu_{d}$ if dust particles are negative, which completely agree with the laboratory experiments [63], [69] on the other hand phase speed decreases with $\mu_{d}$ if dust particles are positive. To verify this prediction, we propose to perform an experiment on the variation of the phase speed with the positive dust number density by using an experimental setup where positive dust can be produced [70].

7. The potential of the solitary wave increases with the increase of the values of $\alpha, \beta_{n}$, and $\mu_{d}$ (if dust particles are positive) but it decreases with the increase of the values of $\mu_{e}$ and $\mu_{d}$ (if dust particles are negative). On the other hand, the width of the solitary waves increases with the increase of the values of $\mu_{e}$ and $\mu_{d}$ (if dust particles are positive) but it decreases with the values of $\mu_{d}$ (if dust particles are positive).

In the present work we have studied nonlinear wave with mK-dV equation. To observe the higher order nonlinearity one can derive modified Zakharov Kuznetsov (mZK) equation [71], Gardner equation [72], [73] which is combined into KdV-mKdV equations, modified Gardner equation [74], Kadomtsov Petviashivilli (KP) equation [75], Gardner KP (GKP) equation [76] and nonlinear Scrhodinger equation [77]. We have intuit the model based on the study of different astrophysical plasma systems like localized electrostatic disturbances in space (viz. auroral plasma, SaturnŠs E- and F-rings, etc.) [27], [78], [79], [80]. However, our present results may be useful for understanding the space [27], [78], [79], [80] and laboratory [27], [69], [81] dusty plasmas. 


\section{References}

1. S. V. Berezhnoj, U. Buddemeier, I. Kaganovich and C. B. Shin, "Charged species profiles in oxygen plasm", Applied Physics Letters, vol. 77, pp. 800-802, 2000.

2. R. N. Franklin, "Electronegative plasmas-why are they so different?", Plasma Sources Science and Technology, vol. 11, pp. A31-A37, 2002.

3. T. Kimura, K. Inagaki and K. Ohe, "The spatial profiles of charged particles in a multipolar magnetically confined Ar- $\mathrm{O}_{2}$ plasma", Journal of Physics D, vol. 31, pp. 2295-2304, 1998.

4. D. Vender, W. W. Stoffels, E. Stoffels, G. M. W. Kroesen and F. J. de Hoog, "Charged-species profiles in electronegative radio-frequency plasmas", Physical Review E, vol. 51, pp. 2436-2444, 1995.

5. G. O. Ludwig, J. L. Ferreira and Y. Nakamura, "Observation of ion-acoustic rarefaction solitons in a multicomponent plasma with negative ions", Physical Review Letters, vol. 52, pp. 275-278, 1984.

6. B. Song, D. Suszcynsky, N. D'Angelo and R. L. Merlino, "Electrostatic ion-cyclotron waves in a plasma with negative ions", Physics of Fluids B, vol. 1, pp. 2316-2318, 1989.

7. T. Intrator, N. Herskowitz and R. Stern, "Beamplasma interactions in a positive ion - negative ion plasma", Physics of Fluids, vol. 26, pp. 1942-1948, 1983.

8. J. A. Johnson III and R. Ramaiah, "Plasma instability in the presence of negative ions", Physics Review A, vol. 36, pp. 774-794, 1987.

9. N. Plihon, C. S. Corr and P. Chabert, "Double layer formation in the expanding region of an inductively coupled electronegative plasma", Applied Physics Letters, vol. 86, pp. 091501-3, 2005.

10. S. H. Kim and R. L. Merlino, "Charging of dust grains in a plasma with negative ions", Physics of Plasmas, vol. 13, pp. 052118, 2006.

11. W. M. Moslem and P. K. Shukla, "Ion thermal double layers in a pair-ion plasma containing charged dust impurities", Physics Letters A, vol. 362, pp. 463-467, 2007.

12. S. Ali, W. M. Moslem and P. K. Shukla, "Wake potential with mobile positive/negative ions in multicomponent dusty plasmas", Physics of Plasmas, vol. 372, pp. 6650-6652, 2008.

13. H. Kokura, S. Yoneda, K. Nakamura, N. Mitsuhira, M. Nakamura and H. Sugai, "Diagnostic of surface wave plasma for oxide etching in comparison with inductive rf plasma", Japanese Journal of Applied Physics, vol. 38, pp. 5256-5261, 1999.

14. L. Boufendi and A. Bouchoule, "Industrial developments of scientific insights in dusty plasmas", Plasma Sources Science and Technology, vol. 11, pp. A211-A218, 2002.

15. M. A. Lieberman and A. J. Lichtenberg, "Principle of Plasma Discharges and Materials Processing", 2nd ed. Wiley, New York, 2005.

16. J. H. Chu, J. B. Du and I. Lin, "Coulomb solids and low-frequency fluctuations in rf dusty plasmas", Journal of Physics D: Applied Physics, vol. 27, pp. 296-300, 1994.

17. J. H. Chu and I. Lin, "Direct observation of coulomb crystals and liquids in strongly coupled rf dusty plasmas", Physical Review Letters, vol. 72, pp. 4009-4012, 1994.

18. H. Thomas, G. E. Morfill, V. Dammel, J. Goree, B. Feuerbacher and D. Möhlmann, "Plasma crystal: coulomb crystallization in a dusty plasma", Physical Review Letters, vol. 73, pp. 652-655, 1994.

19. P. K. Shukla and B. Eliasson, "Colloquium: Fundamentals of dust-plasma interactions", Reviews of Modern Physics, vol. 81, pp. 25-44, 2009.

20. A. A. Mamun, R. A. Cairns and P. K. Shukla, "Dust negative ion acoustic shock waves in a dusty multi-ion plasma", Physics Lettets A, vol. 373, pp. 2355-2359, 2009.

21. M. Rosenberg and R. L. Merlino, "Ion-acoustic instability in a dusty negative ion plasma", Planetary and Space Science, vol. 55, pp. 1464-1469, 2007.

22. R. L. Merlino and S. H. Kim, "Charge neutralization of dust particles in a plasma with negative ions", Applied Physics Letters, vol. 89, pp. 091501-091502, 2006.

23. B. M. Annaratone and J. E. Allen, "A note on the potential acquired by a dust particle in an electronegative plasma", Journal of Physics D: Applied Physics, vol. 38, pp. 26-28, 2005.

24. B. M. Annaratone, T. Antonova, H. M. Thomas and G. E. Morfill, "Diagnostics of the electronegative plasma sheath at low pressures using microparticles", Physics Review Letter, vol. 93, pp. 185001, 2004.

25. N. D'Angelo, "Low-frequency waves in collisional positive dust plasmas", Journal of Physics D: Applied Physics, vol. 37, pp. 860-862, 2004.

26. A. A. Mamun and P. K. Shukla, "Charging of dust grains in a plasma with negative ions", Physics of Plasmas, vol. 10, pp. 1518-1520, 2003.

27. P. K. Shukla and A. A. Mamun, "Introduction to dusty plasma physics", 2002.

28. F. Verheest, "Waves in dusty space plasmas", 2000.

29. G. E. Morfill and A. V. Ivlev, "Complex plasmas: An interdisciplinary research field", Reviews of Modern Physics, vol. 81, pp. 1353-1404, 2009. 
30. S. E. Cousens, S. Sultana, I. Kourakis, V. V. Yaroshenko, F. Verheest, and M. A. Hellberg, "Nonlinear dust-acoustic solitary waves in strongly coupled dusty plasmas", Physical Review E, vol. 86, pp. 066404-066414, 2012.

31. S. K. El-Labany and W. F. El-Taibany, "Effect of dust-charge variation on dust acoustic solitary waves in a dusty plasma with trapped electrons", Journal of Plasma Physics, vol. 70, pp. 69-87, 2004.

32. E. A. Bogdanov and A. A. Kudryavtsev, "The conditions for realization of the Boltzmann distribution of negative ions in a plasma", Technical Physics Letters, vol. 27, pp. 905-907, 2001.

33. R. N. Franklin and J. Snell, "The Boltzmann relation in electronegative plasmas: When is it permissible to use it?", Journal of Plasma Physics, vol. 64, pp. 131-153, 2000.

34. A. J. Lichtenberg, I. G. Kouznetsov, Y. T. Lee, M. A. Lieberman, I. D. Kaganovich and L. D. Tsendin, "Modelling plasma discharges at high electronegativity", Plasma Sources Science and Technology, vol. 6, pp. 437-449, 1997.

35. Y. Ghim, S. H. Kim and N. Hershkowitz, "Experimental verification of Boltzmann equilibrium for negative ions in weakly collisional electronegative plasmas", Applied Physics Letters, vol. 94, pp. 151503-151900, 2009.

36. I. Kourakis and P. K. Shukla, "Lagrangian description of nonlinear dust ion acoustic waves in dusty plasmas", European Physical Journal D, vol. 30, pp. 97-103, 2004.

37. M. G. M. Anowar and A. A. Mamun, "Multi-dimensional instability of dust-ion-acoustic solitary waves in a multi-ion dusty plasma", Journal Plasma Physics, vol. 75, pp. 475-493, 2009.

38. W. M. Moslem and W. F. El-Taibany, "Effect of two-temperature trapped electrons to nonlinear dust-ionacoustic solitons", Physics of Plasmas, vol. 12, pp. 122309-7, 2005.

39. A. A. Mamun P. K. Shukla and B. Eliasson, "Solitary waves and double layers in a dusty electronegative plasma", Physics Review E, vol. 80, pp. 046406-6, 2009.

40. A. A. Mamun, P. K. Shukla and B. Eliasson, "Arbitrary amplitude dust ion-acoustic shock waves in a dusty plasma with positive and negative ions", Physics of Plasmas, vol. 16, pp. 114503, 2009.

41. C. Lin and M. M. Lin, "Analytical study of the dust acoustic waves in a magnetized dusty plasma with many different dust grains", Advanced Studies in Theoretical Physics, vol. 1, pp. 563-570, 2007.

42. C. Lin, W. G. Feng and M. M. Lin, "Communications in nonlinear science and numerical simulation", New York, USA: Elsevier, vol. 13, 2008.

43. A. A. Mamun, "Compressive and rarefactive dust-acoustic solitary struc-tures in a magnetized two-iontemperature dusty plasma", Astrophysics and Space Science, vol. 260, pp. 507-514, 1999.

44. A. A. Mamun, "Coexistence of positive and negative solitary potential structures in dusty plasma", Physics Letter A, vol. 372, pp. 686-689, 2008.

45. X. D. Du, K. Toi, M. Osakabe, S. Ohdachi, T. Ido, K. Tanaka, M. Yokoyama, M. Yoshinuma, K. Ogawa, K. Y. Watanabe, M. Isobe, K. Nagaoka, T. Ozaki, S. Sakakibara, R. Seki, A. Shimizu, Y. Suzuki, H. Tsuchiya, and LHD Experiment Group, "Resistive interchange modes destabilized by helically trapped energetic ions in a helical plasma", Physics Review Letter, vol. 114, pp. 155003, 2015.

46. H. Schamel, "Stationary solitary, snoidal and sinusoidal ion acoustic waves", Plasma Physics, vol. 14, pp. 905-924, 1972.

47. H. Schamel, "Analytic BGK modes and their modulational instability", Journal of Plasma Physics, vol. 13, pp. 139-145, 1975.

48. H. Schamel, "Electron holes, ion holes and double layers", Physics Reports, vol. 140, pp. 161-191, 1986.

49. H. Schamel, "Hole equilibria in Vlasov-Poisson systems: A challenge to wave theories of ideal plasmas", Physics of Plasmas, vol. 7, pp. 4831-4844, 2000.

50. D. Winske, S. P. Gary, M. E. Jones, M. Rosenberg, V. W. Chow and D. A. Mendis, "Ion heating in a dusty plasma due to the dust/ion acoustic instability", Geophysical Research Letters, vol. 22, pp. 2069-2072, 1995.

51. Z. J. Zhou, H. Y. Wang and K. B. Zhang, "Dust-acoustic solitary waves in a dusty plasma with two-temperature nonthermal ions", Pramana Journal of Physics, vol. 78, pp. 127-133, 2012.

52. D. K. Ghosh, P. Chatterjee, P. K. Mandal and B. Sahu, "Nonplanar ion-acoustic shocks in electronÜpositronÜion plasmas: Effect of superthermal electrons",Pramana Journal of Physics, vol. 81, pp. 491-501, 2013.

53. H. Schamel, "A modified Korteweg-de Vries equation for ion acoustic waves due to resonant electrons", Journal of Plasma Physics, vol. 9, pp. 377-387, 1973.

54. A. A. Mamun, R. A. Cairns and P. K. Shukla, "Effects of vortex-like and nonthermal ion distributions on nonlinear dust-acoustic waves", Physics of Plasmas, vol. 3, pp. 2610-2614, 1996.

55. A. A. Mamun, "Solitary waves in a three-component dusty plasma with trapped ions", Physica Scripta, vol. 57, pp. 258-260, 1998.

56. S. K. El-Labany, W. F. El-Taibany, A. A. Mamun and W. M. Moslem, "Dust acoustic solitary waves and double layers in a dusty plasma with two-temperature trapped ions", Physics of Plasmas, vol. 11, pp. 926-933, 2004. 
57. M. G. M. Anowar and A. A. Mamun, "Effects of two-temperature electrons and trapped ions on multidimensional instability of dust-acoustic solitary waves", IEEE Transactions on Plasma Science, vol. 37, pp. 1638-1645, 2009.

58. M. G. M. Anowar, M. S. Rahman and A. A. Mamun, "Nonlinear dust-acoustic waves in a strongly coupled dusty plasma with vortexlike ion distribution", Physics of Plasmas, vol. 16, pp. 053704-5, 2009.

59. O. Rahman and A. A. Mamun, "Dust-ion-acoustic solitary waves in dusty plasma with arbitrarily charged dust and vortex-like electron distribution", Physics of Plasmas, vol. 18, pp. 083703-5, 2011.

60. A. A. Mamun, "Nonlinear propagation of dust-acoustic waves in magnetized a dusty plasma with vortex-like ion distribution", Journal of Plasma Physics, vol. 59, pp. 575-580, 1998.

61. H. Schamel and S. Bujarbarua, "Solitary plasma hole via ion-vortex distribution", Physics of Fluids, vol. 23, pp. 2498-2499, 1980.

62. A. A. Mamun, B. Eliasson and P. K. Shukla, "Dust-acoustic solitary and shock waves in a strongly coupled liquid state dusty plasma with a vortex-like ion distribution", Physics Letter A, vol. 332, pp. 412-416, 2004.

63. H. Washimi and T. Taniuti, "Propagation of ion-acoustic solitary waves of small amplitude", Physics Review Letter, vol. 17, pp. 996-997, 1966.

64. A. A. Mamun, P. K. Shukla and L. Stenflo, "Obliquely propagating electron-acoustic solitary waves", Physics of Plasmas, vol. 9, pp. 1474-1477, 2002.

65. A. A. Mamun, "Nonlinear propagation of ion-acoustic waves in a hot magnetized plasma with vortexlike electron distribution", Physics of Plasmas, vol. 5, pp. 322-324, 1998.

66. X. Qi, Y. X. Xu, W. S. Duan and L. Yang, "The application scope of the reductive perturbation method and the upper limit of the dust acoustic solitary waves in a dusty plasma", Physics of Plasmas, vol. 21, pp. 013702-7, 2014

67. T. Taniuti and C. Wei, "Reductive perturbation method in nonlinear wave propagation I", Journal of the Physical Society of Japan, vol. 24, pp. 941-946, 1968.

68. C. H. Su and C. S. Gardner, "Korteweg-de Vries Equation and Generalizations. III. Derivation of the Korteweg-de Vries Equation and Burgers Equation", Journal of Mathematical Physics, vol. 10, pp. 536-538, 1969.

69. A. Barkan, N. DŠAngelo and R. L. Merlino, "Experiment on ion-acoustic waves in dusty plasmas", Planetary and Space Science, vol. 44, pp. 239-242, 1996.

70. X. Liang, J. Zheng, J. X. Ma, W. D. Liu, J. Xie, G. Zhuang and C. X. Yu, "Experimental observation of ion-acoustic waves in an inhomogeneous dusty plasma", Physics of Plasmas, vol. 8, pp. 1459-1462, 2001.

71. M. M. Haider1, T. Ferdous and S. S. Duha, "Instability due to trapped electrons in magnetized multi-ion dusty plasmas", Journal of Theoretical and Applied Physics, vol. 9, pp. 159-166, 2015.

72. C. Gardner, J. Greene, M. Kruskal and R. Miura, "Method for solving the Korteweg-de Vries equation", Physical Review Letters, vol. 19, pp. 1095- 1097, 1967.

73. A. M. Wazwaz, "New solitons and kink solutions for the Gardner equation", Communications in Nonlinear Science and Numerical Simulation, vol. 12, pp. 1395-1404, 2007.

74. A. A. Mamun and S. Islam, "Nonplanar dust-ion-acoustic double layers in a dusty nonthermal plasma", Journal of Geophysical Research, vol. 116, pp. A12323-6, 2011.

75. B. B. Kadomtsev and V. I. Petviashvili, "On the stability of solitary waves in weakly dispersive media", Soviet Physics Doklady, vol. 15, pp. 539-541, 1970.

76. A. M. Wazwaz, "Solitons and singular solitons for the Gardner-KP equation", Applied Mathematics and Computation, vol. 204, pp. 162-169, 2008.

77. P. E. Zhidkov, "Korteweg-de Vries and nonlinear Schrodinger's equations: qualitative theory", Springer Verlag New York, NY. 2001.

78. M. G. M. Anowar and A. A. Mamun, "Multidimensional instability of electron-acoustic solitary waves in a magnetized plasma with vortex-like electron distribution", Physics of Plasmas, vol. 15, pp. 102111-6, 2008.

79. C. K. Goertz, "Dusty plasmas in the solar system", Reviews of Geophysics, vol. 27, pp. 271-292, 1989.

80. D. A. Mendis and M. Rosenberg, "Cosmic dusty plasma", Annual Review of Astronomy and Astrophysics, vol. 32, pp. 419-463, 1994.

81. R. L. Merlino and J. Goree, "Dusty plasmas in the laboratory, industry, and space", Physics Today, vol. 57, pp. 32-38, 2004. 